\title{
Antimicrobial stewardship in a rural regional hospital - growing a positive culture
}

\author{
E Junaid, ${ }^{1}$ MB ChB, BSc; L Jenkins, ${ }^{2}$ MB ChB, MFamMed, FCFP (SA), PhD; H Swanepoel, ${ }^{3}$ MB ChB, FC Path Clin (SA), MMed (Clin Path); \\ Z North, ${ }^{4} \mathrm{MB}$ ChB, MPH; T Gould, ${ }^{5} \mathrm{MB}$ ChB, MMed (Int Med), FCP (SA) \\ ${ }^{1}$ University of Manchester, UK; and Improving Global Health, NHS Thames Valley and Wessex Leadership Academy, UK \\ ${ }^{2}$ Head of Clinical Unit: Family and Emergency Medicine, George Regional Hospital, Department of Health, Western Cape, South Africa; and \\ Department of Family and Emergency Medicine, Faculty of Medicine and Health Sciences, Stellenbosch University, Cape Town, South Africa \\ ${ }^{3}$ Clinical pathologist, National Health Laboratory Service, George Regional Hospital, Department of Health, Western Cape, South Africa \\ ${ }^{4}$ Medical Manager, George Regional Hospital, Department of Health, Western Cape, South Africa \\ ${ }^{5}$ Head of Clinical Unit: Internal Medicine, George Regional Hospital, Department of Health, Western Cape, South Africa
}

Corresponding author: L Jenkins (louis.jenkins@westerncape.gov.za)

Antimicrobial stewardship programmes have been introduced worldwide in response to the rise in antimicrobial resistance. The World Health Organization has mandated each Member State to produce a plan to address this problem. We report on the organic development of an antibiotic stewardship programme in a rural regional hospital in a resource-limited setting in South Africa. This has resulted in organisational change with increased awareness, participation, monitoring and education in antibiotic stewardship throughout the hospital.

S Afr Med J 2018;108(7):546-550. DOI:10.7196/SAMJ.2018.v108i7.13149

Antimicrobials are used to treat various infections caused by bacteria, viruses, fungi, protozoa and parasites. Of these, antibiotics specifically are used to treat various bacterial infections. Before Alexander Fleming's revolutionary discovery of penicillin, millions of people died of now treatable infectious diseases. However, as he predicted with his discovery, the ability of microbes to develop resistance posed a threat to antibiotic treatment in the future. Fleming stated: '.. there is the danger that the ignorant man may easily under dose himself and by exposing his microbes to nonlethal quantities of the drug make them resistant. ${ }^{[1]}$ Consequently, microbes have become increasingly 'resistant' and survive against the antimicrobials that were once effective to treat infections. One driver of developing resistance is the inappropriate use of antimicrobials.

Antimicrobial resistance is a growing problem worldwide. The increase in antimicrobial resistance, together with the insufficient numbers of new antimicrobials being produced, have triggered global concern about the future of treating infectious diseases. There is a worldwide fear that since the last major class of antibiotics was released in 1987, a post-antibiotic era is coming where there will not be enough agents to treat resistant infections. ${ }^{[2,3]}$ Consequently, the World Health Organization (WHO) introduced the Global Action Plan on Antimicrobial Resistance at the 2015 World Health Assembly, with a focus on antimicrobial stewardship. Antimicrobial stewardship programmes include the appropriate use of antimicrobials, aiming to reduce resistance to antimicrobials and prevent the spread of infection. It was mandated that each Member State of the WHO should produce its own plan. ${ }^{[3]}$

This article describes the development of an institutional antibiotic stewardship programme, with a focus on bacterial infection, based on the national plan and conceptual framework of the National Department of Health and implemented over 3 years in a rural regional hospital in South Africa (SA).

\section{National context}

SA has a high burden of morbidity and mortality from infectious diseases. $^{[4]}$ One study in 2003 (National Department of Health, South Africa, 'Impact of the Essential Drugs Programme at primary health care level in South Africa', described in the 2017 South African Health Review ${ }^{[5]}$ ) showed that the percentage of encounters in which an antibiotic was prescribed increased from $36 \%$ to $47 \%$ between 1998 and 2003. In view of the global strategies for antimicrobial stewardship and the mandate from the WHO, a national plan for antimicrobial stewardship in SA was developed, together with a conceptual framework (Fig. 1). ${ }^{[5]}$

\section{Local context}

George Hospital is a level 2 regional referral hospital with primary, secondary and tertiary services, located in the Western Cape Province of SA. This 272-bed facility has specialists in most clinical departments, operating theatres, outpatient departments, critical care and a robust outreach and support programme to the 10 outlying district hospitals. The pharmacy department consists of six non-ward pharmacists. The on-site National Health Laboratory Service (NHLS) laboratory has a clinical pathologist trained in microbiology, and the hospital has a nursing-based infection prevention and control (IPC) clinical programme co-ordinator. There is a strong commitment to the training of undergraduate and postgraduate students from the universities of Cape Town and Stellenbosch, interns, international students, and students and interns in the nursing, allied health and pharmacy professions. The hospital serves the communities of Eden and Central Karoo districts, which have a combined population of $\sim 685000$ people, with a literacy rate of $82.6 \%$ and $73.4 \%$, respectively. ${ }^{[7,8]}$ The main languages spoken are Afrikaans, English and Xhosa.

Antibiotic stewardship was first introduced in the Western Cape at Groote Schuur Hospital (GSH), which is the referral hospital for 
George, in 2012. ${ }^{[9]}$ In the initial phase of its antibiotic stewardship programme, the GSH Department of Internal Medicine introduced dedicated antibiotic stewardship ward rounds and a dedicated prescription chart. ${ }^{[10]}$ Subsequently, during outreach from the Department of Internal Medicine at GSH to George Hospital, the hospital became sensitised to antibiotic stewardship and started to develop its own local antimicrobial stewardship programme.

\section{The antibiotic stewardship programine}

Early days - incubating the concepts

In February 2015, the Department of Internal Medicine at George Hospital started creating awareness around antibiotic stewardship.

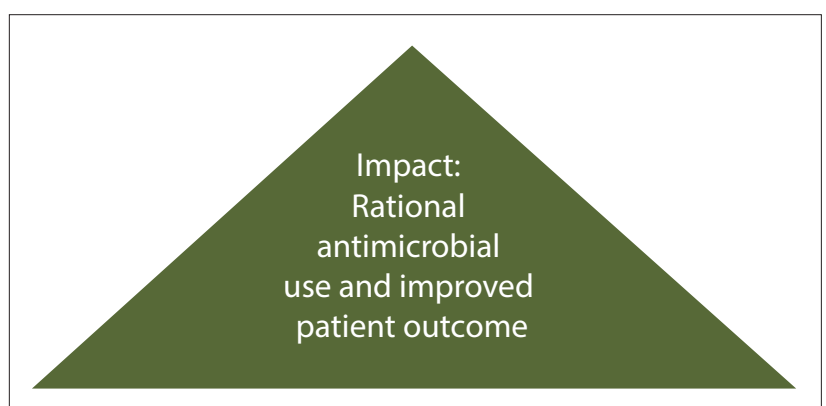

Antimicrobial resistance governance
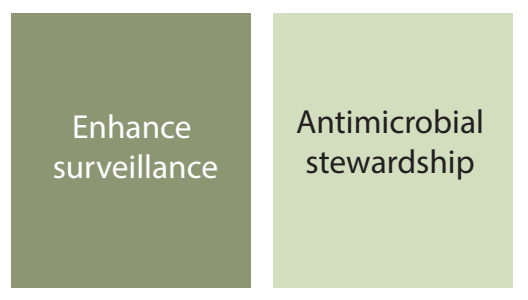

Prevention

including

IPC and

vaccination

Education and communication/public awareness

Health systems strengthening, research, education and communication

Fig. 1. Pillars of the South African antimicrobial stewardship strategy framework. ${ }^{[6]}($ IPC $=$ infection prevention and control. $)$
As part of a collaborative leadership development initiative between the Western Cape Department of Health and the Improving Global Health (IGH) through Leadership Development Programme in the UK, the hospital has been allocated a dedicated young doctor or other health professional for 6-month periods, to partake in a local systems improvement project. Three 'fellows' at a time have been rotating for 6-month periods at George Hospital since 2014, each addressing a specific quality improvement project in the George subdistrict. These fellows are supervised by local senior health professionals, who act as mentors to help the fellows develop leadership skills in a health management environment. With each 6-month period, a fellow is allocated to quality improvement of the antibiotic stewardship programme. This has ensured continued local leadership by a dedicated champion for antibiotic stewardship improvement over 3 years, with each subsequent fellow continuing where the previous fellow left off. We utilised project management principles from the quality improvement model used by Brink et al.$^{[11]}$ in an antibiotic stewardship implementation study in 47 SA hospitals, which ran over 5 years.

At the start of this collaboration, there was no formal antimicrobial stewardship programme. Antibiotic stewardship ward rounds were being held in three departments, namely General Surgery, Paediatrics and Internal Medicine. With ongoing outreach support from the tertiary hospital (GSH), and learning from their programme, George Hospital introduced an antibiotic stewardship programme in February 2015. This included several components, namely:

- Weekly dedicated antibiotic stewardship ward rounds, attended by multi-professional teams of doctors, pharmacists and nurses and the clinical pathologist from the on-site NHLS laboratory

- A dedicated prescription chart

- Audit tools for pharmacy, IPC and ward rounds

- Regular multi-professional reviews with doctors, pharmacists, nurses, the clinical pathologist, management and the IGH fellow

- A hospital-wide education programme incorporating current principles of antibiotic stewardship, with small-group discussions, posters, flash cards, and an e-training module for staff (Table 1) ${ }^{[10]}$

- Infection prevention and control programme monitoring.

Standards were set for 6-monthly audits and were adjusted upon review during each successive phase of the programme, relevant to and integrated with the performance of the hospital at the time. Implementation data were collected by the antibiotic stewardship audit team consisting of the current IGH fellow, the IPC nurse, the pharmacy and the clinical pathologist. Data included uptake of the antibiotic stewardship ward rounds in each clinical department, antibiotic consumption, quality of prescribing, use of laboratory tests and antibiotic chart compliance.

\section{Table 1. Checklist for optimal antibiotic prescribing ${ }^{[12]}$}

1. Drug - which is the narrowest-spectrum antibiotic that I can use to treat this bacterial infection?

2. Dose - many antibiotics require weight-based dosing and their dosing depends on renal and/or hepatic function.

3. Dose frequency - dependent on the half-life of the drug and whether the action of the antibiotic depends on the time above the MIC or the area under the concentration/time curve. Calculation of the dosing frequency may require therapeutic drug monitoring, such as for vancomycin or aminoglycosides

4. Duration - should be dictated by evidence from randomised controlled trials whenever possible. Expert opinion from national and international guidelines should be consulted where evidence is weak.

5. Route - most antibiotics have good oral bioavailability, but some infections will require intravenous therapy either for the whole or part of the course.

6. De-escalation - applies to the spectrum of antibiotic use and route of administration. All attempts to convert early from parenteral to oral use should be made.

MIC = minimum inhibitory concentration. 


\section{Results - growing the culture}

The main findings of the programme so far have been the following:

\section{Increased awareness among staff of antibiotic} stewardship principles

This has been achieved through various media, including posters that explain correct infection prevention and control practices and address issues of hand washing and isolation procedures for the different indications, as well as posters on principles for antibiotic prescribing. While only the Department of Internal Medicine engaged with the programme in 2015, roll-out and uptake of the programme across all clinical departments has resulted in a changed institutional culture over 3 years; for example, the newly introduced antibiotic prescription charts are now integrated into the general prescription charts across the hospital.

Institution-wide uptake of antibiotic stewardship ward rounds Dedicated weekly multi-professional ward rounds are taking place in the departments of General Surgery, Internal Medicine, Family Medicine, Paediatrics, Emergency Medicine, Orthopaedics, Neonatology and Obstetrics and Gynaecology. The number of ward rounds has increased; for example, in February 2017 there were only four rounds, which increased to 25 in November 2017.

\section{Increased tracking and reporting in antibiotic} stewardship process measurements

Since the start of the programme, antibiotic prescription chart auditing has been ongoing and continually evolving, as has the production of data on antibiotic consumption, antibiotic stewardship ward round quality, intravenous line and trans-urethral catheter use, and healthcare-associated infections. These data are now being reported to staff at all levels through email, via a dedicated noticeboard and an electronic communication system and at the Hospital Antimicrobial Stewardship Committee meetings. Antibiotic consumption data are collected and analysed provincially through a system of defined daily doses (DDDs)/100 days and sent through to the hospital on a periodic basis (Fig. 2).

Comprehensive completion of the prescription chart, including patient weight, allergies, renal function and proof of culture, is a way to show that there has been consideration of all parameters related to antibiotic prescribing. It also allows for pharmacists to assess the chart when it is reviewed by them. An antibiotic prescription chart audit in November 2017 showed that dosing considerations filled in on the chart have improved for weight and estimated glomerular filtration rate (eGFR); however, allergy entries decreased compared with January 2017 (weight 88\%, allergy 72\%, eGFR 83\% v. weight $71 \%$, allergy $86 \%$, eGFR $65 \%$, respectively). This has led to increased

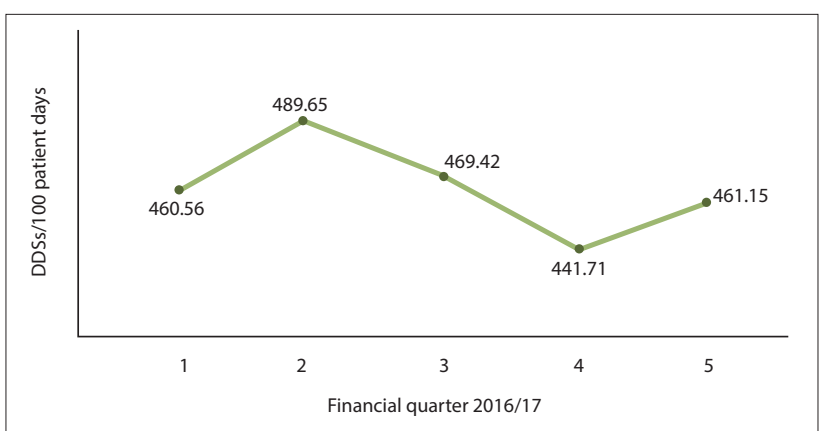

Fig. 2. DDDs/100 patient days at George Hospital between April 2016 and June 2017. (DDDs = defined daily doses.) staff training by the stewardship teams during ward rounds as well as performance reporting at antibiotic stewardship board feedback meetings. Education on sending of cultures prior to antibiotic commencement has resulted in an observed increase in awareness of the principle.

\section{Regular, iterative, patient-centred bedside educational meetings} during ward rounds on antibiotic stewardship

These occur between members of the team and are multi-professional in teaching and learning roles. Members of the rounds report increased knowledge on stewardship principles because of these learning opportunities (reports from other staff members).

\section{Emergence of the multi-professional team during the dedicated ward rounds}

Ward rounds now regularly include the ward doctors, the ward nurses, a pharmacist, the clinical pathologist and the institutional infection control nurse, as well as the IGH fellow.

\section{Dedication of resources towards the programme}

Resources include providing training opportunities in antibiotic stewardship and the allocation of dedicated IGH fellows.

\section{Discussion}

The implementation of the antibiotic stewardship programme at George Hospital has been multifaceted, with various interventions being introduced to strengthen it. The main results have been an increase in awareness of antibiotic stewardship principles and an increase in dedicated ward rounds. These can be attributed to the various components of the programme as described in the National Quality Partners Playbook: Antibiotic Stewardship in Acute Care: ${ }^{[13]}$ leadership commitment, health worker accountability, drug expertise, action, tracking, reporting and education.

Implementing a stewardship programme requires dedicated staff to champion the programme at various levels and in multiple health professions. Antimicrobial stewardship has been championed by the senior management staff at George Hospital, including the provision of four dedicated IGH fellows to the programme, specifically focusing on quality improvement in health systems, the presence of the medical manager on the board of the Hospital Antimicrobial Stewardship Committee, promoting regular training and education opportunities in antibiotic stewardship, and dedicated weekly time allocation to encourage staff to participate in antibiotic stewardship ward rounds.

Overall leadership of the programme itself has been owned by the resident clinical pathologist. The importance of leadership accountability been shown through co-ordination and championing of antibiotic stewardship ward rounds, ensuring that they take place, and education on antibiotic stewardship as an expert in the area. Actively encouraging staff to engage with the rounds on a ward-to-ward basis has been a driving force in increasing buy-in from staff and is now established practice, even in the absence of the IGH fellows.

A number of other quality improvement projects formed part of the programme. For example, an audit was conducted on the appropriate use of surgical prophylaxis. In the orthopaedic ward, intravenous cefazolin was being used as ward stock with little discretionary control. ${ }^{[14]}$ As a result, it was removed as ward stock to enable review of antibiotics prior to dispensing, leading to improved quality of prescriptions in that ward. In addition, auditing of ward round compliance and quality occurred, leading to improvements.

Tracking of metrics in antibiotic stewardship has been produced locally and provincially in the form of a facility antibiogram and 
DDDs, respectively. Local resistance patterns of organisms to antibiotics are collected and presented to the clinical and management staff by the clinical pathologist (Table 2). These are produced according to guidelines as set out by the Clinical and Laboratory Standards Institute, USA. There may be numerous reasons for the lack of change in the DDDs, one of which could simply be the early nature of the programme. It is postulated that the DDDs should have decreased on review in a year's time.

Reporting the hospital's performance in metrics for antibiotic stewardship has been a strong focus. The platforms for reporting have included the Hospital Antimicrobial Stewardship Committee meetings held on a quarterly basis, where data are presented, feedback is given and areas for improvement are proposed. This information is made available to all hospital staff through the hospital's online enterprise content management platform. The information is also displayed through a dedicated noticeboard in antibiotic stewardship. These platforms all provide ways to raise awareness of what is being done for stewardship in the hospital and why it is being done, and encourage staff to identify areas for improvement and formulate recommendations.

Education in antibiotic stewardship principles has also been a key component of the programme. This has included face-to-face training of pharmacy staff, doctors and nurses at the patient bedside during ward rounds, as well as a George Hospital-specific online learning module for annual training to be completed during the induction programme by new medical staff. Posters have also been distributed around the hospital to educate the wider non-clinical medical staff and the public, particularly in conjunction with the annual World Antibiotic Awareness Week. The development of signage for infection prevention and control processes, which includes isolation of patients with communicable diseases, particularly tuberculosis, and handhygiene instruction posters, has helped to educate the public and other non-medical staff.

The commencement of the multiprofessional stewardship rounds across all departments and the strengthening of the previously commenced ward rounds have added a new level of patient-centred care around evidencebased medicine, with the various team

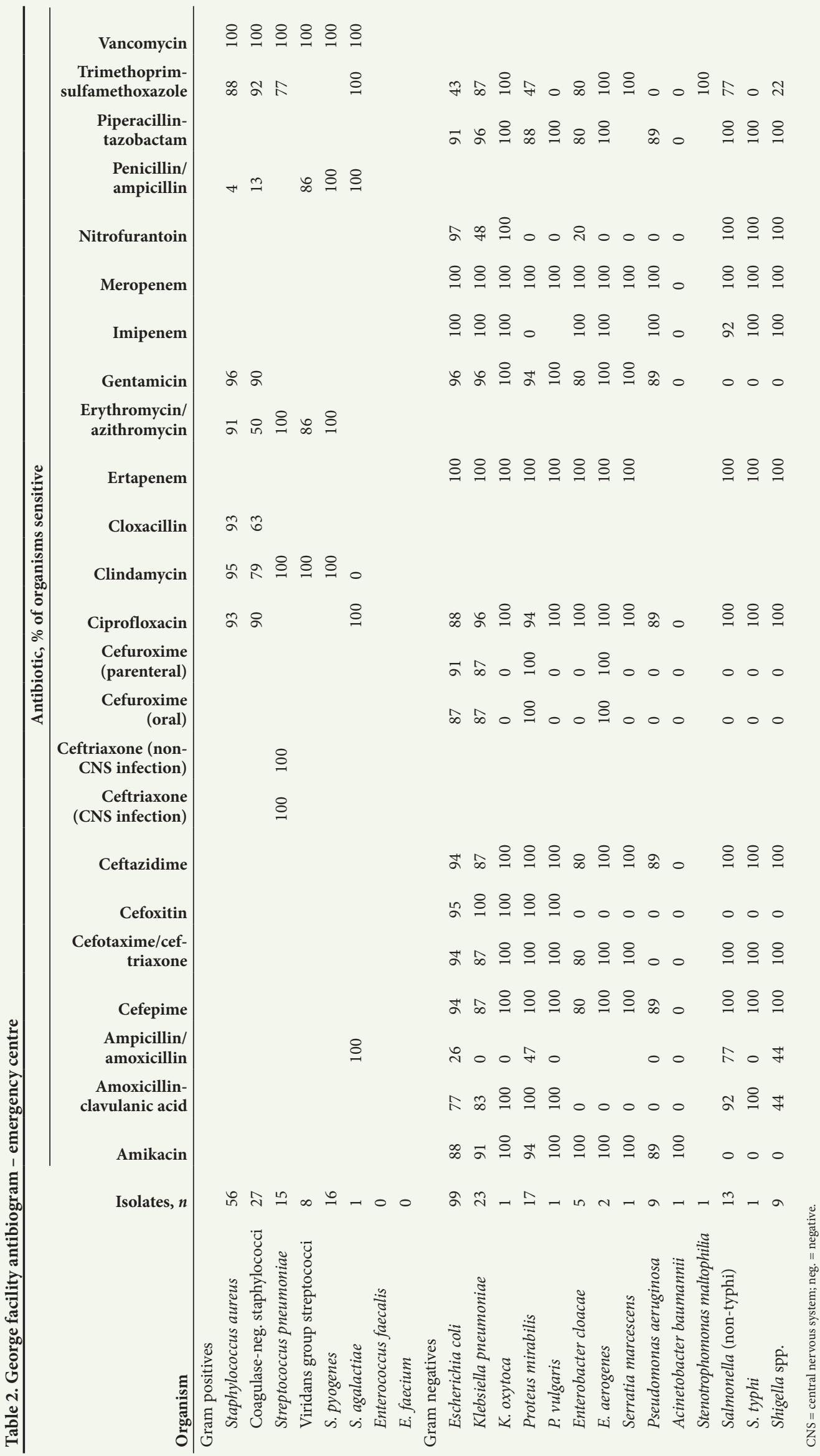


members discussing and learning together, which we have observed to encourage communication between disciplines, checking the Essential Drugs List, the provincial coding list and the South African Antibiotic Stewardship Programme mobile application for accurate and appropriate prescriptions. ${ }^{[14,15]}$

\section{Future growth}

Antibiotic stewardship is continually evolving worldwide. Before 2015, George Hospital had no explicit awareness of or structured approach to antibiotic stewardship. While the hospital is situated in a public, rural, resource-limited setting, a robust quality improvement programme in antibiotic stewardship has been initiated and is continually being improved. This article attempts to share this local experience with a wider audience.

Future goals include producing antibiograms for the six surrounding subdistricts to engage and increase the understanding of staff in the referring hospitals around antibiotic stewardship, and recognising the effect of micro-organism resistance in referring hospitals in Eden and Central Karoo. Other goals include the introduction of a dedicated pharmacist who is trained in and has expertise in antibiotic stewardship. Local prescription guidelines based on local resistance patterns will also be developed. Auditing and reporting should continue to improve and expand, considering the further use of information technologies in antibiotic stewardship metrics. Similarly, online resources and other media forums for the local communities for antibiotic education could be of benefit. The key, as described by Mendelson, ${ }^{[12]}$ is for '.. every prescriber to become an antibiotic steward'.

Acknowledgements. The authors are grateful for the support of all the staff at George Hospital who are still participating in the programme. Support for the programme from the CEO, Mr M Vonk, the nursing head, Ms E Sellars, the head of pharmacy, Ms S Brits, and Eden District Office is also acknowledged.

Author contributions. EJ conceptualised the original manuscript. LJ and HS contributed extensively to the subsequent drafts. All authors added to and approved the final draft.
Funding. None.

Conflicts of interest. None.

1. Fleming A. Fleming Lecture. Nobel Prize. 11 December 1945. https://www.nobelprize.org/nobel_prizes/ medicine/laureates/1945/fleming-lecture.pdf (accessed 27 January 2018).

2. World Health Organization. Global Action Plan on Antimicrobial Resistance. 2015. http://apps.who.int iris/bitstream/10665/193736/1/9789241509763_eng.pdf?ua=1 (accessed 27 January 2018).

3. O'Neill J. Review on Antimicrobial Resistance. Tackling drug-resistant infections globally: Final report and recommendations. May 2016. https://amr-review.org/sites/default/files/160518_Final\%20paper_ and recommendations. May 2016. https://am.
with\%20cover.pdf (accessed 27 January 2018).

4. Statistics South Africa. Mortality and causes of death in South Africa, 2015: Findings from death

Statistics South Africa. Mortality and causes of death in South Africa, 2015: Findings from death
notification. http://www.statssa.gov.za/publications/P03093/P030932015.pdf (accessed 27 January 2018) notification. http://www.statssa.gov.za/publications/P03093/P030932015.pdf (accessed 27 January 2018),
Padarath A, Barron P, eds. South African Health Review 2017. Durban: Health Systems Trust:53. Padarath A, Barron P, eds. South African Health Review 2017. Durban: Health Systems Trust:53,
http://www.hst.org.za/publications/South\%20African\%20Health\%20Reviews/HST\%20SAHR\%20 2017\%20Web\%20Version.pdf (accessed 6 June 2018)

6. National Department of Health, South Africa. Antimicrobial Resistance National Strategy Framework 2014 - 2024. Pretoria: $\mathrm{NDoH}, 2014$. http://www.health.gov.za (accessed 27 January 2018).

7. Western Cape Government. Socio-economic Profile Central Karoo District Municipality. Cape Town: Western Cape Government, 2016. https://www.westerncape.gov.za/assets/departments/ treasury/Documents/Socio-economic-profiles/2016/Central-Karoo-District/dc05_central_karoo district_2016_socio-economic_profile_sep-lg.pdf (accessed 27 January 2018).

8. Western Cape Government. Socio-economic Profile Eden District Municipality. Cape Town: Western Cape Government, 2016. https://www.westerncape.gov.za/assets/departments/treasury/Documents/ Socio-economic-profiles/2016/Eden-District/dc04_eden_district_municipality_2016_socioeconomic profile_sep-lg.pdf (accessed 27 January 2018).

9. Boyles TH, Whitelaw A, Bamford C, Moodley M, Bonorchis K. Antibiotic stewardship ward round ayd a dedicated prescription chart reduce antibiotic consumption and pharmacy costs without
and and a dedicated prescription chart reduce antibiotic consumption and pharmacy costs without
affecting inpatient mortality or re-admission rates. PLoS One 2013;8(12). https://doi.org/10.1371/ journal.pone.0079747

10. Federation of Infectious Diseases Societies of Southern Africa. About SAASP: Mission Statement of the South African Antibiotic Stewardship Programme. 2017. http://www.fidssa.co.za/SAASP (accessed 14 December 2017)

11. Brink AJ, Messina AP, Feldman C, et al. Antimicrobial stewardship across 47 South African hospitals An implementation study. Lancet Infect Dis 2016;16(9):1017-1025. https://doi.org/10.1016/S14733099(16)30012-3

12. Mendelson M. Role of antibiotic stewardship in extending the age of modern medicine. S Afr Med J 2015;105(5):414-418. https://doi.org/10.7196/SAMJ.9635

13. National Quality Forum. Antibiotic Stewardship in Acute Care. Washington, DC: The Joint Commission, 2016. https://www.jointcommission.org/assets/1/6/New_Antimicrobial_Stewardship_ Standard.pdf (accessed 27 January 2018).

14. National Department of Health, South Africa. Standard Treatment Guidelines and Essential Medicines National Department of Health, South Africa. Standard Treatment Guidelines and Essential Medicines
List for South Africa. 2015. http://www.kznhealth.gov.za/pharmacy/hospitallevel_adult2015.pdf (accessed 27 January 2018).

15. Wasserman S, Boyles T, Mendelson M. South African Antibiotic Stewardship Programme - a Pocket Guide to Antibiotic Prescribing for Adults in South Africa. https://www.iidssa.co.za/Content/ Documents/SAASP_Antibiotic_Guidelines_2015.pdf (accessed 27 January 2018).

Accepted 10 May 2018 